Received: 03.08 .2018

Revised: 07.05.2020

Accepted: 19.06 .2020

DOI: $10.17804 / 2410-9908.2020 .3 .019-028$

\title{
DETERMINATION OF HYDROGEN-INDUCED DAMAGE BY AN ACOUSTIC METHOD
}

\author{
V. V. Mishakin ${ }^{\text {a)* }}$, A. V. Gonchar ${ }^{\text {b) }}$, A. V. Poroshkov, N. V. Semenova \\ Mechanical Engineering Research Institute - Branch of the Federal Research Center \\ Institute of Applied Physics of the RAS, 85 Belinskogo St., Nizhny Novgorod, 603024, Russian Federation \\ a) (iD https://orcid.org/0000-0002-4329-7938 $\otimes$ imndt31@mts-nn.ru; \\ b) iDhttps://orcid.org/0000-0001-9408-4206 \\ *Corresponding author. E-mail: imndt31@mts-nn.ru \\ Address for correspondence: 85 Belinskogo St., Nizhny Novgorod, 603024, Russia
}

The 13HFA corrosion- and cold-resistant steel is studied by an ultrasonic method after hydrogen absorption for 96, 192, and 288 hours. Within the framework of classical flaw detection, it was found that the formation of macrodefects occurs only at 288 hours of hydrogen absorption. In the study of the material by the spectral-acoustic method, it was found that, during hydrogen absorption for 96 and 192 hours, the signal spectrum changes. This indicates the formation of microdefects. It is shown that the determination of the damage by the conventional ultrasonic flaw detection method and the use of the spectral-acoustic method for the evaluation of fracture at the microlevel give more complete information on the state of the investigated alloys.

Keywords: hydrogen absorption, hydrogen-induced damage, acoustic method, spectral-acoustic method, attenuation.

\section{Acknowledgment}

The study was performed under the state assignment for IAP RAS, theme No. 0035-20140402.

\section{References}

1. Melnikov A.V., Misharin D.A., Bogdanov R.I., Ryakhovsky I.V. Justification of the health of gas pipelines with stress corrosion cracking defects. Korroziya Territorii Neftegaz, 2015, vol. 31, no. 2, pp. 32-40. (In Russian).

2. Brondel D., Edwards R., Hayman A., Hill D., Mehta S., Semerad T. Corrosion in the oil industry. Oilfield Review, 1994, vol. 6, no. 2, pp. 4-18.

3. Gafarov N.A., Mitrofanov A.V., Goncharov A.A., Tret'yak A.Ya., Kichenko B.V. The analysis of the equipment and pipeline damages in extracting processing and transportation in Orenburg Oil and Gas Plant. IRTs Gazprom. Ser. Diag. Oborud. i Trubopr., 2000, pp. 1-40. (In Russian).

4. $\quad$ Krдgeloh J., Wolfgang B. Einfluss der Eigenspannung auf die Sauergasbestdndigkeit Hoch Frequenz Induktiv geschweiЯter Rohre unter Berbcksichtigung prozessspezifischer Einflussfaktoren, Shaker, 2009, 164 p.

5. NACE TM0284-2011, Evaluation of Pipeline and Pressure Vessel Steels for Resistance to Hydrogen-induced Cracking, NACE International, Houston, TX, 2011.

6. Truell R., Elbaum C., Chick B.B. Ultrasonic Methods in Solid State Physics, Academic Press, New York and London, 1969. 
7. Klyuev V.V., ed., Nerazrushayushchiy control: Spravochnik v 7 t. [Nondestructive testing. A Handbook in 7 Vols.]. Moscow, Mashinostroenie, 2004.

8. Adler L., Rose J. H., and Mobley C. Ultrasonic method to determine gas porosity in aluminum alloy castings: Theory and experiment. J. Appl. Phys., 1986, vol. 59, iss. 2, pp. 336-347. DOI: $10.1063 / 1.336689$.

9. Mishakin V.V., Naumov M.Y., Mishakin S.V., Kassina N.V. Russian Journal of Nondestructive Testing, 2007, vol. 43, pp. 677-682. DOI: 10.1134/S1061830907100075.

10. Rose J.H. Ultrasonic characterization of porosity: Theory. Review of Progress in Quantitative Nondestructive Evaluation, vol. 4B, D. O. Thompson and D. E. Chimenti, eds., Plenum, New York, 1985, p. 909. 
Подана в журнал: 03.08.2018

УДК 620.179.(147+162)

DOI: $10.17804 / 2410-9908.2020 .3 .019-028$

\title{
ОПРЕДЕЛЕНИЕ ИНДУЦИРОВАННОЙ ВОДОРОДОМ ПОВРЕЖДЕННОСТИ АКУСТИЧЕСКИМ МЕТОДОМ
}

\author{
В. В. Мишакин ${ }^{\text {a)* }}$, А. В. Гончар ${ }^{\text {б) }}$, А. В. Порошков, Н. В. Семенова \\ Институт проблем машиностроения РАН - филиал ФГБНУ \\ «Федеральный исследовательский иентр Институт прикладной физики РАН», \\ 85, Белинского, г. Нижний Новгород, Россия. \\ a) iDhttps://orcid.org/0000-0002-4329-7938 imndt31@mts-nn.ru; \\ б) iDhttps://orcid.org/0000-0001-9408-4206
}

\begin{abstract}
*Ответственный автор. Электронная почта: imndt31@ @ts-nn.ru;
Адрес для переписки: ул. Белинского, 85, 603024, г. Нижний Новгород, Российская Федерация
\end{abstract}

Проведено исследование ультразвуковым методом коррозионностойкой и хладостойкой стали 13ХФА после наводороживания 96 ч, 192 ч и 288 ч. В рамках классической дефектоскопии установлено, что образование макродефектов происходит только при максимальном времени наводороживания (288 ч). При исследовании материала спектральноакустическим методом установлено, что в образцах, обработанных сероводородом 96 ч и 192 ч, устойчиво регистристрируется изменение спектра сигнала, что свидетельствует об образовании микродефектов.

Показано, что определение поврежденности традиционным методом ультразвуковой дефектоскопии и использование спектрально-акустического метода для оценки разрушения на микроуровне дает более полную информацию о состояниях исследуемых сплавов.

Ключевые слова: наводороживание, индуцированная водородом поврежденность, акустический метод, спектрально-акустический метод, затухание.

\section{1. Введение}

Поврежденность, индуцированная водородом, - один из основных факторов разрушения магистральных трубопроводов [1-3]. Низкий энергетический барьер для проникновения атома водорода в подповерхностную зону $\mathrm{Fe}$, высокая подвижность водорода в $\alpha$-железе $(1 \times 10-4 c \mathrm{c} 2 \mathrm{c}-1)$, по сравнению с углеродом и азотом $(1 \times 10-16 \mathrm{~cm} 2 \mathrm{c}-1)$ [4], приводит к быстрому увеличению его концентрации, объединению в молекулы в ловушках, в качестве которых могут быть, например, строчечные включения. Высокое давление, создаваемое после объединения атомов водорода, приводит к локальному разрушению материала.

Существует методика оценки склонности металла к водородному растрескиванию с помощью ультразвукового контроля [5]. Расчет поврежденности по этой методике заключается в сравнении суммарной площади несплошностей, образованных в результате водородного повреждения, с общей исследуемой площадью. Определение такого вида повреждений относится к методу традиционной ультразвуковой дефектоскопии, т. е. поиску отражений сигналов от макродефектов и нахождению по этим отражениям эффективных размеров и координат залегания дефектов. Такой подход не охватывает дефекты, которые имеют размеры, близкие к длине волны, или существенно меньшие размеры (длинноволновое приближение).

Существует значительное количество работ о том, что затухание ультразвука используется для определения структурного состояния в длинноволновом приближении, например: 
оценка характеристик дислокационной структуры (модель Гранато-Люка) [6], диаметра зерна [7], поврежденности, связанной с пористостью материала [8] и др.

Ранние исследования показали, что использование таких акустических характеристик, как параметр акустической анизотропии и частотно-зависимого затухания, позволяет оценить величину накопленной поврежденности металлических сплавов на ранней стадии разрушения при статическом и циклическом деформировании [9].

Во многих поликристаллических материалах все вышеперечисленные факторы присутствуют одновременно. Описание материала с такой системой дефектов, в том числе математическое описание, представляет значительные трудности.

Индуцированная водородом поврежденность имеет ряд особенностей по сравнению с поврежденностью, полученной в результате силового нагружения. Существенного изменения геометрии объектов при поврежденности водородом не происходит. Внедрение атомов водорода в материал не может привести к заметному изменению характеристик кристаллографической текстуры, повышению плотности дислокаций. В основном, объединение в молекулы в ловушках, высокое давление, создаваемое после объединения атомов водорода, образование гидридов приводят к локальному разрушению, появлению микропор и микротрещин, а при дальнейшем наводороживании - к появлению макродефектов - расслоений.

Ослабление живого сечения за счет образования несплошностей широко используется для расчета прочности и долговечности материалов. Оценочные расчеты размеров дефектов и их концентрации неразрушающими методами контроля более полно определяют состояния материалов силовых элементов конструкций.

При использовании контактных пьезоэлектрических преобразователей (ПЭП) затухание связано с дифракционными потерями, рассеянием на элементах структуры, поглощением, определяемым вязкостью материала, рассеянием на внутренних и внешних поверхностях при вводе и приеме УЗ-волн, потерей в контактном слое, потерей части энергии в ПЭП.

С учетом дифракционных потерь и затухания в материале амплитуду сигнала $A$, прошедшего расстояние $X$, при условии, что расстояние $X$ больше размера ближней зоны, можно записать как [7]:

$$
A=A_{0} * 2\left|\operatorname{Sin} \frac{\pi a^{2}}{2 \lambda X}\right| e^{-X \alpha_{\tilde{n} \partial े},}
$$

где $\alpha_{\text {стр. }}-$ затухание, связанное со структурой материала; $A_{0}-$ амплитуда сигнала при $X=0$; $\lambda$ - длина волны.

Измеряя амплитуду первого $A_{1}$ и второго $A_{2}$ отраженных сигналов при использовании эхо импульсного метода из уравнения (1), получим:

$$
\alpha_{\tilde{n} 0 \delta .}(\omega)=\frac{1}{X_{2}-X_{1}}\left(\ln \frac{A_{2}(\omega)}{A_{1}(\omega)}-\ln \left(\sin \left(\frac{a^{2} \omega}{V X_{1}}\right) / \sin \left(\frac{a^{2} \omega}{V X_{2}}\right)\right),\right.
$$

где $\omega$ - циклическая частота; $V$ - скорость упругих волн; $X_{1}$ и $X_{2}$ длина акустического пути первого и второго отраженных сигналов соответственно.

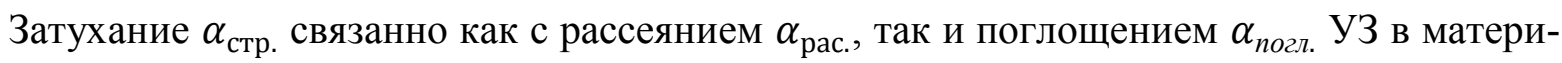
але. 


$$
\alpha_{c m p .}=\alpha_{p a c .}+\alpha_{\text {погл. }}
$$

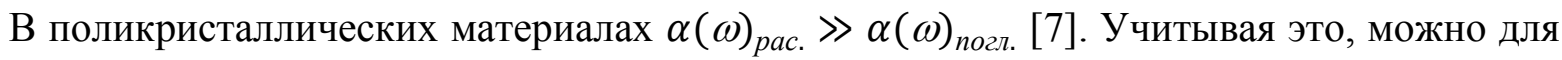
изменения затухания записать:

$$
\Delta \alpha \approx \Delta \alpha(\omega)_{p a c}
$$

Разделяют затухание, связанное с рассеянием на границах зерен и порах [10]:

$$
\alpha_{\text {pac. }}=\alpha_{p}+\alpha_{\mathrm{g}}
$$

где $\alpha_{p}$ - затухание на порах $\left(\alpha_{p} \sim f^{2}\right) ; \alpha_{g}$ - затухание на границах зерен $\left(\alpha_{g} \sim f^{4}\right)$.

При исследовании использовали длинноволновое приближение, когда длина волны $\lambda$ больше диаметра зерна или поры.

Для спектра сигнала $S$ получим:

$$
S=S_{0} * 2\left|\operatorname{Sin} \frac{\pi a^{2}}{2 \lambda X}\right|,
$$

где $S_{0}$-амплитудно-частотный спектр сигнала при $X=0$. на $X$.

Как видно из выражений, на спектр сигнала существенное влияние оказывает величи-

Составляющую затухания $\alpha_{p}$ можно определить, не вводя поправки на потери, связанные с дифракцией, например измеряя амплитуду первого отраженного сигнала до и после появления водородоиндуцированной поврежденности.

Образование водородоиндуцированной поврежденности не приводит к существенным изменениям длины акустического пути $X$ и, следовательно, не приводит к изменению дифракционных потерь. В этом случае изменение затухания в зонах материала, где длина волны существенно больше размерных характеристик элементов структуры, связано в основном с образованием и накоплением микропор, микротрещин.

Для материала с рассеянием на границах зерен и материала с рассеянием упругих волн на границах и порах с учетом дифракционной поправки можно записать:

$$
\begin{gathered}
\frac{A_{g}}{A_{0}}=2\left|\operatorname{Sin} \frac{\pi a^{2}}{2 \lambda X_{n}}\right| \exp \left[X_{n}\left(-\alpha_{g}\right)\right] ; \\
\frac{A_{p g}}{A_{0}}=2\left|\operatorname{Sin} \frac{\pi a^{2}}{2 \lambda X_{n}}\right| \exp \left[X_{n}\left(-\alpha_{g}-\alpha_{p}\right)\right],
\end{gathered}
$$

где $A_{g}$ и $A_{p g}$ - амплитуды сигналов при отражениях на границах зерен (не обработанный образец) и на границах зерен и порах (образцы после обработки) соответственно, $X_{n}-$ длина акустического пути $n$-ого отраженного сигнала.

Исходя из выражений $(7,8)$, составляющую затухания $\alpha_{p}$ можно определить как: 


$$
\alpha_{p}\left(k, X_{n}\right)=\frac{1}{X_{n}} \ln \left[\frac{A_{g}\left(k, X_{n}\right)}{A_{p g}\left(k, X_{n}\right)}\right],
$$

где $k=\omega / V=2 \pi f / V=2 \pi / \lambda-$ волновое число; $f$ - частота; $\lambda-$ длина волны.

Амплитудно-частотный спектр сигналов, прошедших материал без повреждений $A_{g}\left(k, X_{n}\right)$ и материал после обработки $A_{p g}\left(k, X_{n}\right)$, позволяет определить частотно зависимое затухание, связанное с образованием микронесплошностей при водородоиндуцированной поврежденности.

Объемное содержание пор $C$ определяется согласно [8] как:

$$
\begin{gathered}
C=\frac{4}{3}\left[\alpha_{p}(k) \mathrm{a}_{p} / \tilde{A}\left(k \mathrm{a}_{p}\right)\right], \\
\Gamma\left(k a_{p}\right)=\beta(\eta) k^{4} \mathrm{a}_{p}^{4},
\end{gathered}
$$

где $\eta$ - отношение скорости распространения поперечной к продольной волне; $\mathrm{a}_{p}-$ радиус пор.

Для слабо легированных сталей, например для стали 13 ХАА, $\eta=0,53$. Для исследуемого материала при $\eta=0,53-\beta(\eta) \approx 2,1[8]$.

На частотно зависимое затухание влияет весь спектр микродефектов. Определение функции распределения дефектов по размерам только по частотно-зависимому затуханию невозможно. Однако на основе спектрально-акустических измерений можно предложить оценочную характеристику, отражающую рост объемного содержания микронесплошностей.

Предполагая, что наибольшее влияние на затухание оказывают поры, размеры которых приближаются к длине волны $d=2 a_{p} \approx \lambda / 2$, принимая во внимание, что скорость продольной волны $V \approx 6000$ м/с, и, учитывая зависимости (10) и (11), получим:

$$
C \approx 0,03 * \alpha_{p}^{\max } \lambda
$$

где $\alpha_{p}^{\max }$ - максимальное значение затухания, определяемое по кривой частотно-зависимого затухания.

\section{2. Методика}

Образцы для исследования индуцированной водородом поврежденности были изготовлены из стали 13ХФА, которая используется для производства нефтегазопроводных труб повышенной коррозионной стойкости и хладостойкости. Плоскопараллельные образцы прямоугольного сечения размерами $70 \times 30 \times 11$ вырезанные из одного темплета, подвергались воздействию сероводорода в растворе по методике приведенной [5]. Один образец не подвергался воздействию сероводорода. Три образца находились в растворе 96, 192 и 288 ч соответственно.

Для измерения акустических параметров использовалась портативная акустическая установка, состоящая из генератора зондирующих сигналов, высокочастотного усилителя, цифрового осциллографа PC SCOPE 1000VS и ПК. Используемый метод - эхо-импульсный. Применялся высокочувствительный ультразвуковой пьезоэлектрический преобразователь V110 Olympus продольных упругих волн: центральная частота 5 МГц, диаметр преобразо- 
вателя $~ 6$ мм. Рабочая область частот находится в диапазоне от 3 МГц до 9 МГц. Регистрировалась амплитудно-временная диаграмма первого и второго отраженных сигналов. Погрешность измерения времени распространения УВ - около 2-3 нс. Длительность ультразвуковых эхо-импульсов продольных волн - около 0,8 мкс. Погрешность измерения затухания $1 \mathrm{H} \Pi / \mathrm{M}$.

Поиск дефектов производился ультразвуковым датчиком продольных волн фирмы Kraut Kramer с центральной частотой 10 МГц. Размер выявляемых дефектов - 1 мм и более.

На рис. 1 показана схема проведения ультразвуковых исследований. Образец условно был разделен на 8 зон, в которых проводились ультразвуковые исследования.

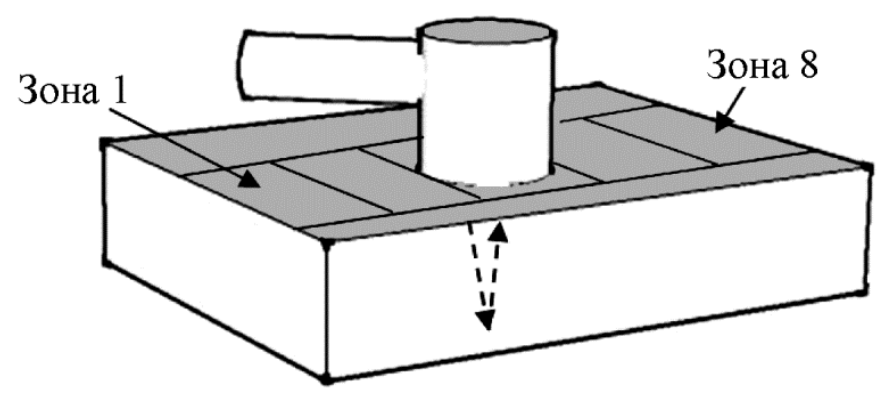

Рис. 1. Схема проведения ультразвуковых исследований

Изменения скорости распространения упругих продольных волн V выше погрешности ее измерения при наводороживании зарегистрировано не было. Для продольной волны она составляла около $5930 \mathrm{~m} / \mathrm{c}$. Значение $A_{g}\left(k, X_{n}\right)$ определялось путем выделения второго отраженного сигнала на образце, не подверженном водородному повреждению, а $A_{p g}\left(k, X_{n}\right)-$ на образцах с водородным повреждением и последующим Фурье преобразованием сигнала для определения зависимости амплитуды от частоты.

Расчет объемного содержания пор проводился следующим образом. Строили зависимость $\alpha_{p}(f)$ по формуле (9), определяли максимальные значения затухания $\alpha_{p}^{\text {max }}$ и соответствующую максимальному затуханию частоту, исходя из которой рассчитывали длину волны и по формуле (11) рассчитывали величину объемного содержания пор.

\section{3. Результаты и обсуждение}

В результате исследования образцов были выявлены зоны, в которых наблюдались отражения от отдельных макродефектов (размеры дефектов $d$ больше длины волны $\lambda$, условно $d>\lambda, \lambda=1,0$ мм) и зоны, в которых отражения от отдельных дефектов не наблюдались $(d<\lambda)$, но затухание существенно изменилось. При наводороживании 96 и 192 ч макродефекты практически не образуются. При наводороживании 288 ч происходит интенсивное образование дефектов. Площадь, занимаемая дефектами $S$, выявляемая ультразвуковым методом, резко увеличивается (рис. 2). 


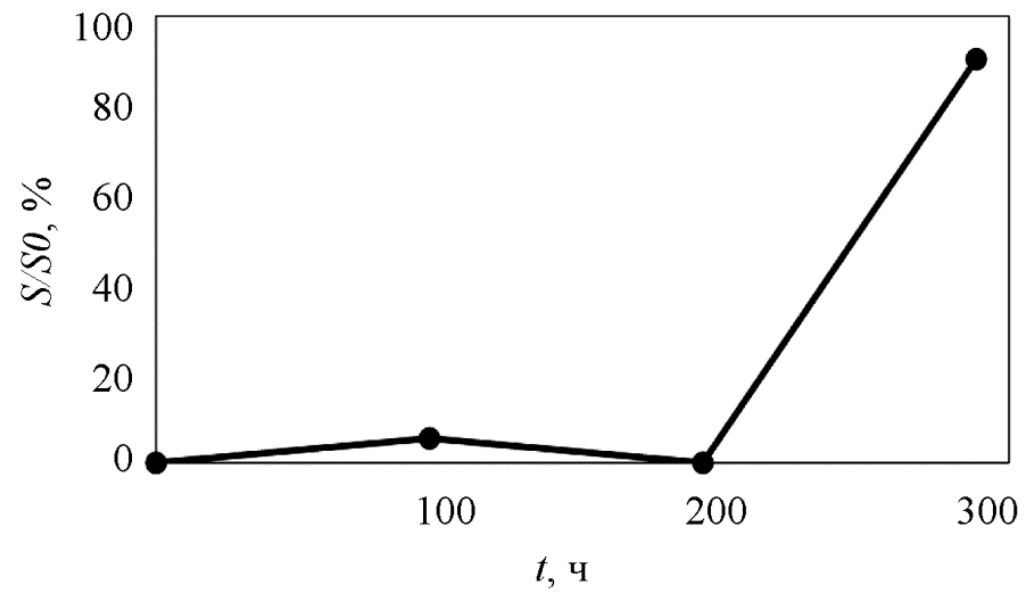

Рис. 2. Площадь, занимаемая дефектами, относительно общей площади исследования $S 0$ в зависимости от времени наводораживания $t$

Наличие микро- и макродефектов подтверждено металлографическими исследованиями микрошлифов торцевой поверхности образцов в продольном направлении. На рис. 2 представлены поры и трещины, вызванные водородным насыщением. Выявленные микродефекты имели как округлую, так и вытянутую форму (рис. $3 a$ ). Дефекты, достигшие больших размеров, имели вытянутую форму (рис. 3 б).

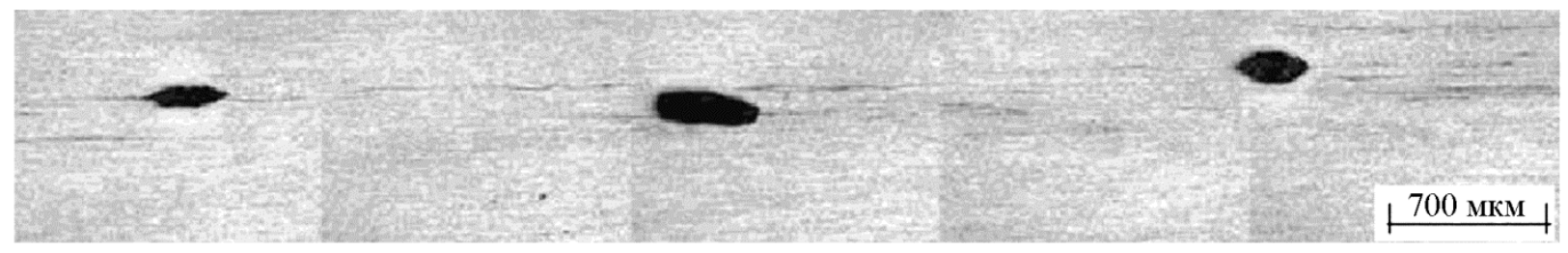

$a$
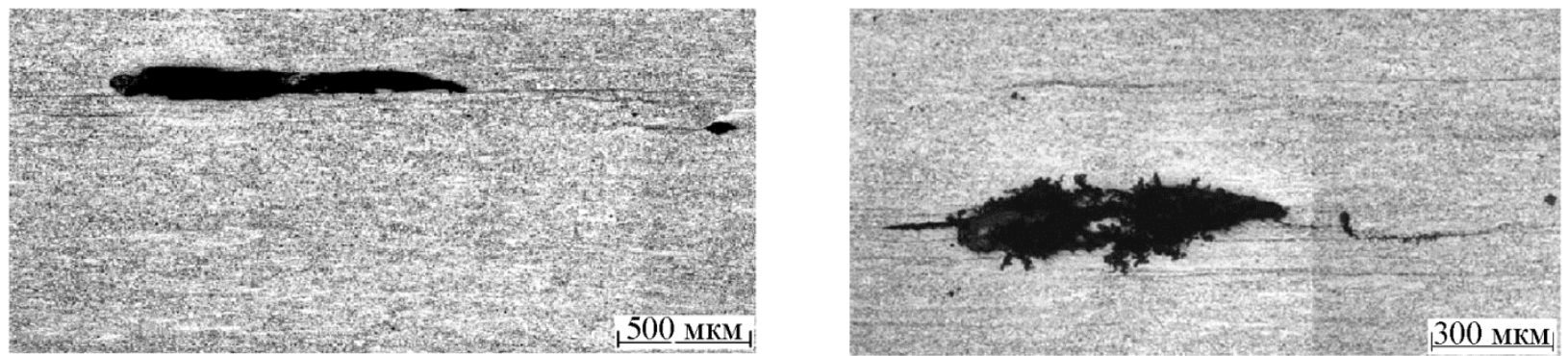

$\sigma$

Рис. 3. Дефекты, образовавшиеся в результате наводороживания образцов (288 ч)

На рис. 4 представлено частотно-зависимое затухание $\alpha_{p}(f)$ для трех образцов с разным временем наводораживания. Затухание рассчитывалось по формуле (9) в зонах, в которых не наблюдались отражения от макродефектов. 


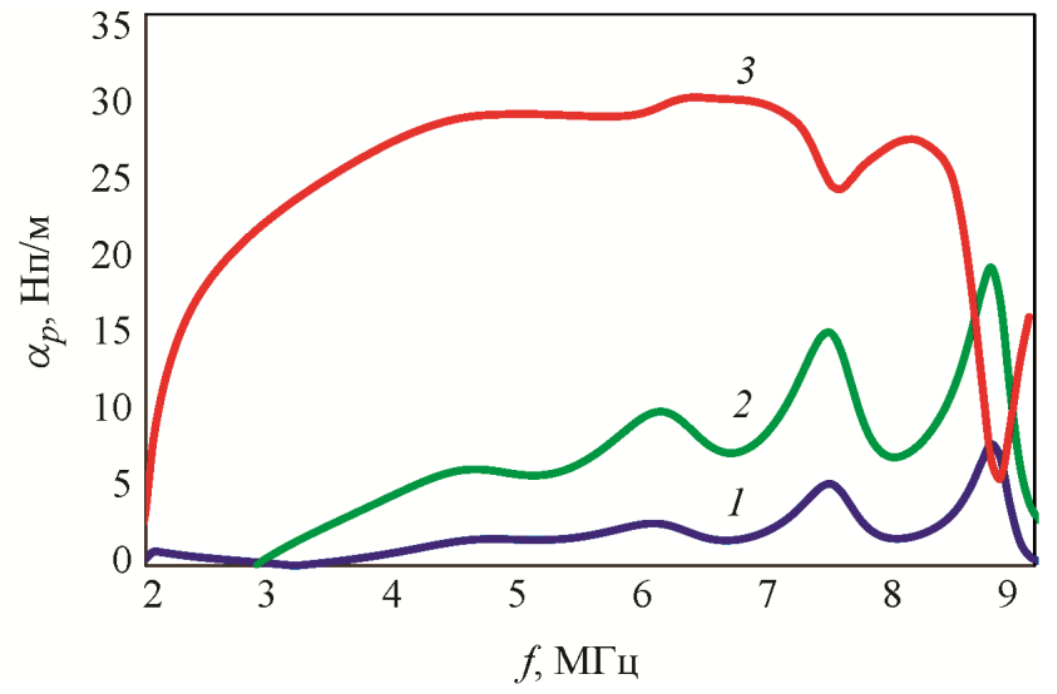

Рис. 4. Зависимость затухания $\alpha_{p}$ от частоты: $1-96$ ч; $2-192$ ч; $3-288$ ч.

Из графика видно, что для образца, обработанного 96 ч, максимальное значение затухания $\alpha_{p}$ составило 6,7 Нп/м на частоте 8,9 МГц, для образца, обработанного 192 ч - 17 Нп/м на частоте 8,7 МГц, для 288 ч - 30 Нп/м на частоте 6,5 МГц.

По формуле (11) используя данные, представленные на рис. 3, можно оценить объемное содержания пор $C$ в зависимости от времени наводороживания (рис. 5).

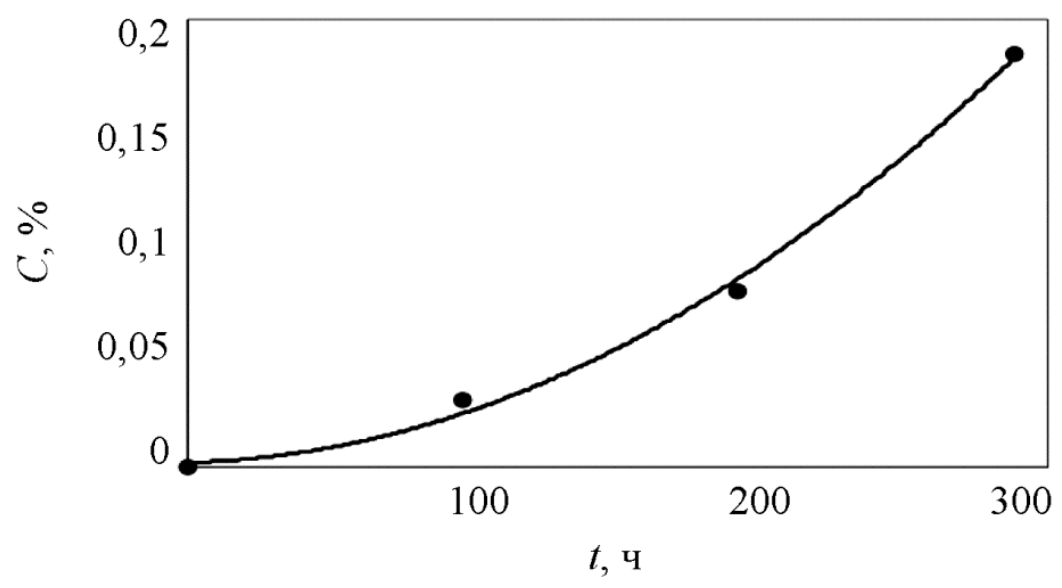

Рис. 5. Зависимость объемного содержания пор $C$ от времени наводороживания

Согласно рис.2, при времени обработки 96 и 192 ч отражений от отдельных макродефектов практически не наблюдается. При спектрально-акустических измерениях в зонах, где не обнаружены макродефекты, исследование показало заметное изменение затухания ультразвука и наличие роста объемного содержания пор.

Для более корректного определения состояния материала уровень его разрушения необходимо осуществлять на микро- и макроуровне.

Можно сказать, что приближение значения параметра $C$ к величине 0,08 \% служит индикатором массового появления макродефектов, существенно влияющих на прочность и живучесть материала конструкции. 


\section{4. Выводы}

Существующая методика ультразвукового контроля поврежденного водородом материала не в полной мере характеризует процесс разрушения сплавов. Такой подход не охватывает дефекты, которые существенно влияют на прочностные характеристики и имеют размеры, меньшие (длинноволновое приближение) или близкие к длине волны.

На примере стали 13ХФА, широко используемой для изготовления нефтегазопроводных труб, приведен алгоритм оценки состояния материала на микро- и на макроуровне.

Показано, что определение поврежденности традиционным методом ультразвуковой дефектоскопии и использование спектрально-акустического метода для оценки разрушения на микроуровне дает более полную информацию о состоянии исследуемых сплавов.

\section{Благодарность}

\section{ИПФ РАН.}

Исследование выполнено в рамках государственного задания по теме № 0035-2014-0402

\section{Литература}

1. Обоснование работоспособности магистральных газопроводов с дефектами коррозионного растрескивания под напряжением / А. В. Мельникова, Д. А. Мишарин, Р. И. Богданов, И. В. Ряховский // Коррозия территории нефтегаз. - 2015. - Т. 31, № 2. - С. 32-40.

2. Corrosion in the oil industry / D. Brondel, R. Edwards, A. Hayman, D. Hill, S. Mehta, T. Semerad // Oilfield Review. - 1994. - Vol. 6, no. 2. - P. 4-18.

3. Анализ повреждений оборудования и трубопроводов на объектах добычи, переработки и транспорта продукции Оренбургского НГКМ / Н. А. Гафаров, А. В. Митрофанов, А. А. Гончаров, А. Я. Третьяк, Б. В. Киченко // ИРЦ Газпром. Серия: диагностика оборудования и трубопроводов. - 2000. - С. 1-40.

4. Krдgeloh J., Wolfgang B. Einfluss der Eigenspannung auf die Sauergasbestzndigkeit Hoch Frequenz Induktiv geschweiЯter Rohre unter Berscksichtigung prozessspezifischer Einflussfaktoren. - Shaker, 2009. - $164 \mathrm{p}$.

5. NACE TM0284-2011. Evaluation of Pipeline and Pressure Vessel Steels for Resistance to Hydrogen-induced Cracking. - NACE International, Houston, TX, 2011.

6. Труэлл Р., Эльбаум Ч., Чик Б. Ультразвуковые методы в физике твердого тела / пер. с англ. - М. : Мир, 1972.

7. Неразрушающий контроль : в 7 т. / под ред. В. В. Клюева. - М. : Машиностроение, 2004. - $736 \mathrm{p}$.

8. Laszlo Adler, Rose James H., Mobley Carroll J. Ultrasonic method to determine gas porosity in aluminum alloy castings: Theory and experiment // Appl. Phys. - 1986. - Vol. 59, no. 2. P. 335-347. - DOI: 10.1063/1.336689.

9. Mishakin V. V., Naumov M. Y., Mishakin S. V., Kassina N. V. Russian Journal of Nondestructive Testing. - 2007. - Vol. 43. - P. 677-682. - DOI: 10.1134/S1061830907100075.

10. Rose J. H. Ultrasonic characterization of porosity: Theory // Review of Progress in Quantitative Nondestructive Evaluation : Vol. 4B / ed. by D. O. Thompson and D. E. Chimenti. - New York : Plenum, 1985. - P. 909. 